Utah State University

DigitalCommons@USU

$1-1-2002$

\title{
Off-Axis Response Measurement of the Sounding of the Atmosphere Using Broadband Emission Radiometry (SABER) Telescope
}
J. L. Stauder
L. R. Bates
J. S. Dyer
R. W. Esplin
D. O. Miles

Follow this and additional works at: https://digitalcommons.usu.edu/sdl_pubs

\section{Recommended Citation}

Stauder, J. L.; Bates, L. R.; Dyer, J. S.; Esplin, R. W.; and Miles, D. O., "Off-Axis Response Measurement of the Sounding of the Atmosphere Using Broadband Emission Radiometry (SABER) Telescope" (2002).

Space Dynamics Lab Publications. Paper 122.

https://digitalcommons.usu.edu/sdl_pubs/122

This Article is brought to you for free and open access by the Space Dynamics Lab at DigitalCommons@USU. It has been accepted for inclusion in Space Dynamics Lab Publications by an authorized administrator of DigitalCommons@USU. For more information, please contact digitalcommons@usu.edu.

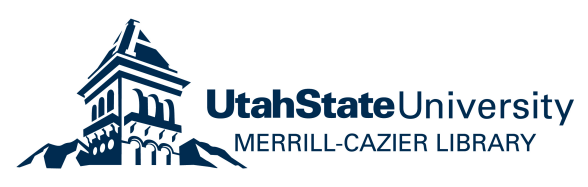




\title{
Off-axis response measurement of the Sounding of the Atmosphere using Broadband Emission Radiometry (SABER) telescope
}

\author{
J.L. Stauder*, L.R. Bates, J.S. Dyer, R.W. Esplin, and D.O. Miles \\ Space Dynamics Laboratory, Utah State University, Logan, UT 84321
}

\begin{abstract}
The Sounding of the Atmosphere using Broadband Emission Radiometry (SABER) instrument is a 10-channel earth limb-viewing sensor that measures atmospheric emissions in the spectral range of $1.27 \mu \mathrm{m}$ to $16.9 \mu \mathrm{m}$. SABER is part of NASA's Thermosphere-Ionosphere-Mesosphere Energetics and Dynamics (TIMED) mission, which was successfully launched in December 2001. Uncommon among limb-viewing sensors, SABER employs an on-axis telescope design with reimaging optics to allow for an intermediate field stop and a Lyot stop. Additional stray light protection is achieved by an innovative inner Lyot stop, which is placed conjugate to the secondary obscuration and support structure. Presented in this paper is the off-axis response of SABER as measured in the Terrestrial Black Hole off-axis scatter facility at the Space Dynamics Laboratory. The measurement was made at visible wavelengths; thus, the response is only representative of SABER's short wavelength channels. The measurement validated the stray light design and complemented the APART software model, which predicts that mirror scatter is the dominant stray light mechanism at short wavelengths. In addition, estimates of the mirror bi-directional reflectance distribution function (BRDF) were made. The off-axis response measurement indicates that SABER is an exceptional stray light suppression telescope.
\end{abstract}

Keywords: off-axis response, stray light, Lyot stop, APART, BRDF, SABER, TIMED

\section{INTRODUCTION}

The Sounding of the Atmosphere using Broadband Emission Radiometry (SABER) instrument is part of the NASA Thermosphere-Ionosphere-Mesosphere Energetics and Dynamics (TIMED) mission, which was placed in a $625 \mathrm{~km}$ orbit on December 7, 2001. The sensor is a 10-channel earth limb-viewing sensor that measures atmospheric emissions in the spectral range of $1.27 \mu \mathrm{m}$ to $16.9 \mu \mathrm{m}$. The telescope, shown in Figure 1, is an on-axis Cassegrain design with a picketfence tuning fork chopper at the first focus. The clamshell re-imager allows for the use of an intermediate field stop and a Lyot stop, which are critical to stray light suppression. A single axis scan mirror is used to obtain vertical profiles of the earth's atmosphere. The instrument description and mission objectives are described in greater detail elsewhere ${ }^{1,2}$. Stray light analyses performed using the software programs APART ${ }^{3}$ and $\mathrm{ASAP}^{4}$ have also been documented ${ }^{5-7}$. The results of the off-axis response measurement of SABER are presented in this report.

Measuring the off-axis response of a telescope characterizes its stray light performance, validates the design, and provides a crosscheck with the software analysis. The response of SABER at visible wavelengths was made in September 1998 at the Space Dynamics Laboratory (SDL) of Utah State University. The resulting off-axis response is compared to an APART model conducted at $0.4 \mu \mathrm{m}$, which is the peak response of the visible detector. This comparison will provide verification of the general APART model for the short wavelength channels of SABER and will help interpret the measured bi-directional reflectance distribution function (BRDF) data of the scan and fore-optics mirrors.

\section{OFF-AXIS MEASUREMENT FACILITY}

The off-axis measurement facility at SDL is often referred to as the terrestrial black hole, and has been described previously by Kemp ${ }^{8}$. Entrance to the room requires the passage through an air shower, which removes loose particles from the occupant. The walls, ceiling, and other components of the room are coated with a diffuse black paint to absorb and scatter incident light. The floor is covered with black carpet, whose fibers are resistant to detachment. The measurement setup located inside the room is depicted in Figure 2. Major components include the specular chamber, HEPA filters (not shown), visible source with collimator, light trap, rotary table, and photo-multiplier tube (PMT). The

* stauder@sdl.usu.edu; phone 1435 797-4388 


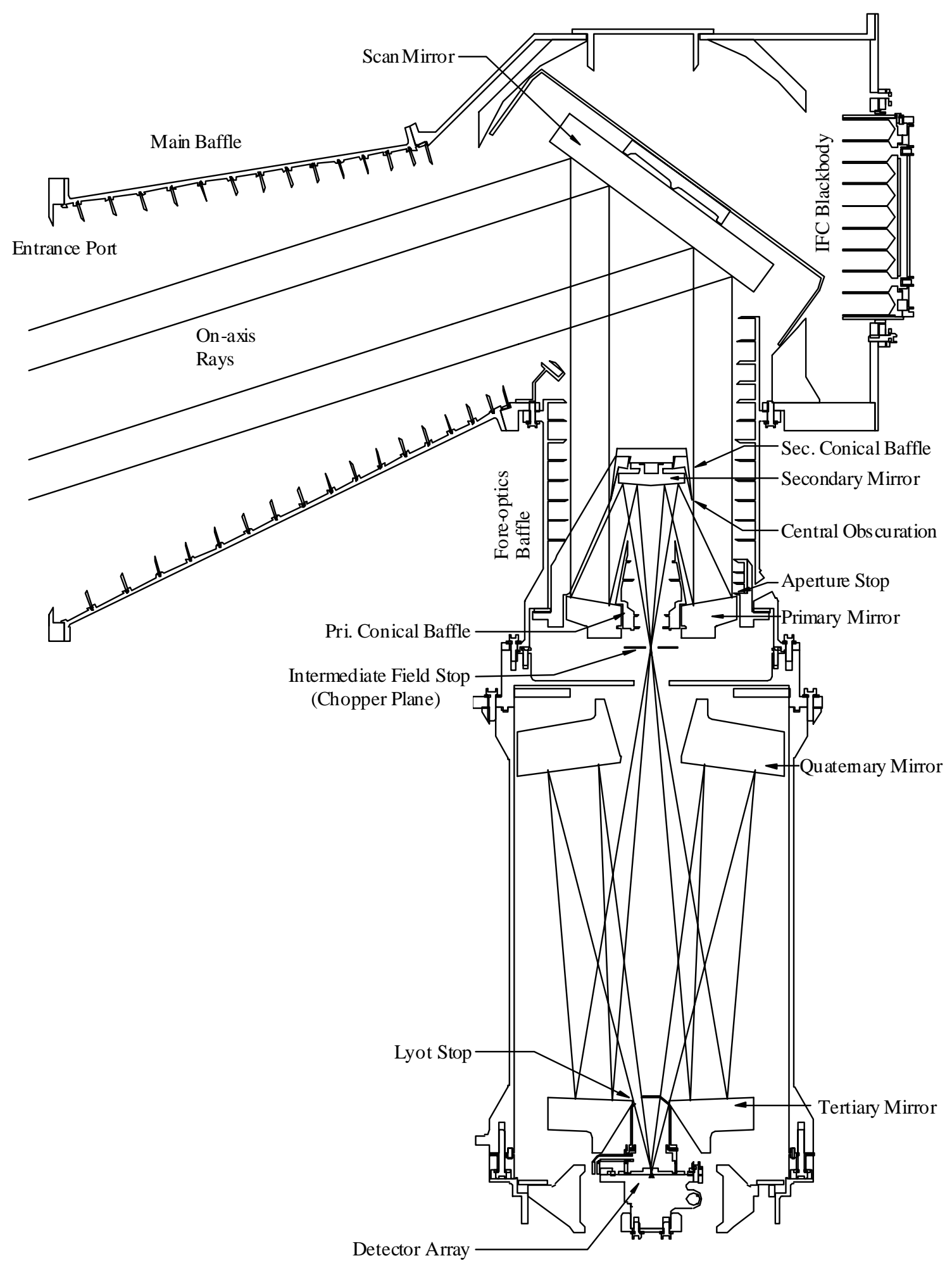

Figure 1. The SABER telescope. 


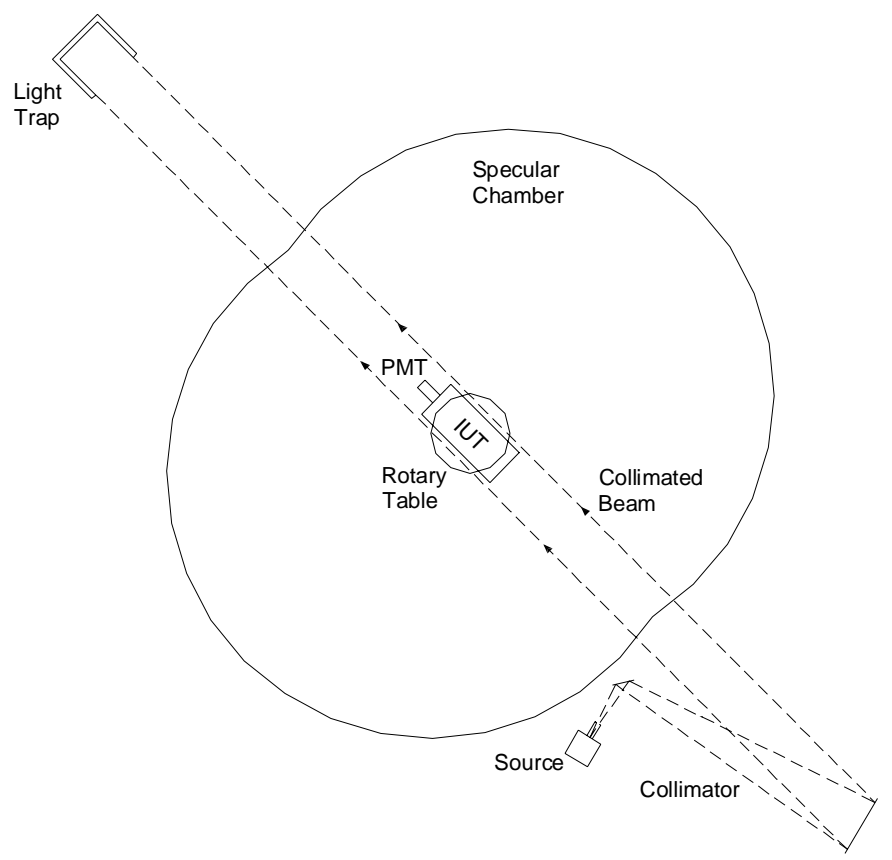

Figure 2. Floor plan of the off-axis measurement facility.

instrument under test (IUT) is mounted on the centrally located rotary table. The eleven-element HEPA filter assembly is placed above the chamber to establish a clean room environment. The filtered air is forced into the chamber from above and exits at floor level beneath the raised chamber walls. Once outside the chamber it returns to the filters, creating a circular airflow. Inside the chamber, a class 100 clean room environment is maintained.

The collimated light, which represents radiation from a distant source, enters the chamber through a 10-inch hole and completely fills the aperture of the IUT. The off-axis response is obtained by incrementally rotating the IUT and recording the power on the focal plane. Light that does not intersect the instrument exits the chamber where it is trapped. The chamber walls redirect light that is scattered off the IUT so that it does not directly reenter the telescope aperture (see Figure 3). A ray loses energy each time it encounters a wall surface. After several reflections, the light rays are reduced to negligible levels.

The rotary table, source, and PMT are controlled from outside of the room via appropriate wires and cables. The visible source is a forced-air-cooled tungsten halogen lamp whose intensity is set by a variable power supply. The lamp is placed at the focus of the collimating optics. The diameter and intensity of the collimated beam can be modified to some extent by changing the aperture size in front of the enclosed lamp. The high precision rotary table is controlled by software running on a UNIX workstation. Visible light is collected by a photon counting PMT detector that requires a 5-volt current-limited power supply. The PMT is commanded and photon counts are read via a serial cable connected to a personal computer.

\section{OFF-AXIS MEASUREMENT OF SABER}

The off-axis response measurement and preparation of SABER was conducted according to detailed procedures given in a pre-defined test plan ${ }^{9}$. In order to simplify the measurement and make the results more understandable, a composite circular field stop was substituted for the picket-fence chopper. At the focal plane, a conjugate circular aperture was mounted in front of the visible detector. SABER's filters and detectors were not installed in the instrument. Once the instrument was mounted onto the rotary table platform, a purge tube was connected to minimize contamination. The offaxis source was aligned to SABER by visually adjusting the source platform and rotary table angular position until the point source was imaged at the center of the focal plane aperture. The PMT was mated to the instrument and measurements were ready to be taken. See Figures $4 \mathrm{a}$ and $4 \mathrm{~b}$. 


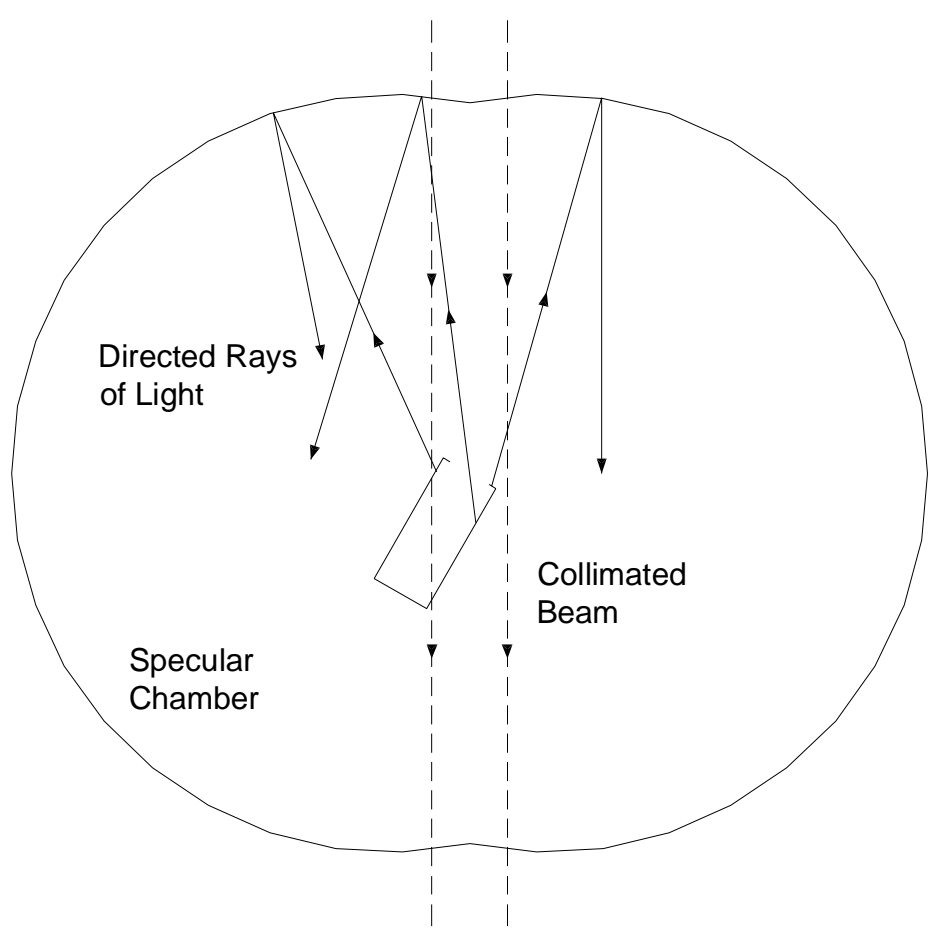

Figure 3. Simple ray trace of specular chamber.
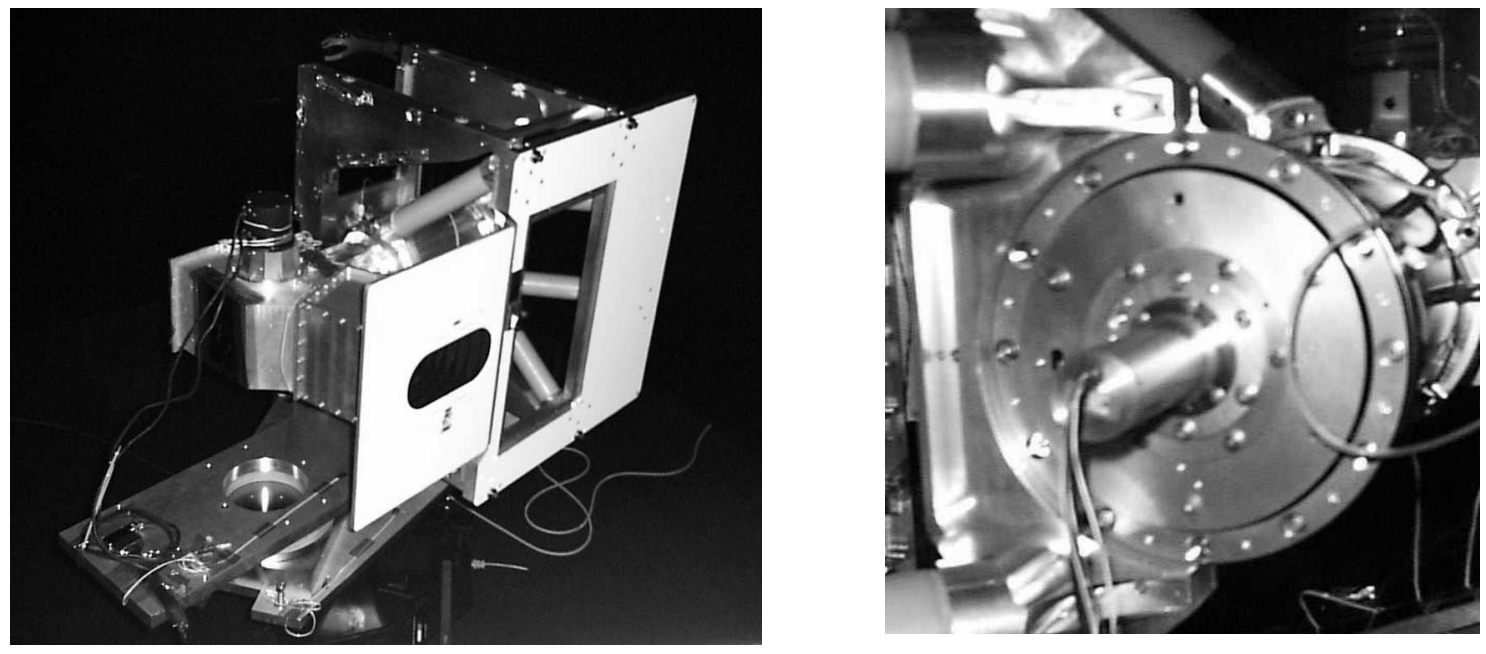

Figure 4. a) SABER on rotary table. b) PMT mated to focal plane assembly. 
The off-axis response is presented in the peak-normalized form of the point source transmission (PST) function. It is defined as the power on the detector, as a function of off-axis source angle, normalized by the detector power due to the source on-axis. A total of 13 data sets were taken of SABER under various instrument configurations. The most tested profile (8 data sets) represents SABER looking at a tangent height of $400 \mathrm{~km}$. Here, the optical axis is coincident with the main baffle axis. The responses taken by the first and last data sets of this configuration are plotted in Figure 5 . Several improvements to the setup were made between these two measurements. The source's collimating mirror was cleaned, and the aperture limiting the cone of light from the pinhole was reduced. This resulted in a lower response at angles between $1.5^{\circ}$ and $3.5^{\circ}$. Initially, the cone of light coming from the source overfilled the collimating mirror. Scattered light from the mirror mounting structure was significant. Even with the source improvements, scatter from the collimating mirror contaminated the response at angles less than 3 degrees. The response was improved at larger angles by covering the front white panel of SABER with a black felt cloth. In the early measurements, a significant amount of scattered light from the panel was scattered again off the chamber walls and re-entered SABER's entrance port. Between $3.5^{\circ}$ and $19^{\circ}$ the response data is consistent between the initial and final measurements. Notice that the off-axis response measurement spanned nearly 11 orders of magnitude, demonstrating excellent stray light control of the setup.

During SABER's mission, vertical profiles of the atmosphere will be obtained by rotating the scan mirror. In the Black Hole, the scan mirror is controlled remotely via a cable passing through the access panel. The instrument response was measured for scan mirror positions corresponding to atmospheric tangent heights of $40 \mathrm{~km}, 70 \mathrm{~km}$, and $400 \mathrm{~km}$. Figure 6 shows the effect on the response as the scan mirror is rotated. Not surprisingly, as the tangent height is decreased, the more effective the shielding of the baffle becomes. Furthermore, the view factor between the scan mirror and the illuminated portion of the baffle is reduced at the lower tangent height mirror positions. This effect has been witnessed on orbit.

The off-axis response measurement of SABER validated a key stray light design feature. The Lyot stop configuration was altered for the final two response measurements to demonstrate the effectiveness of the inner stop and its properly placed supports ${ }^{10}$. The results are shown in Figure 7, where the response labeled "Flight Lyot Stop" is shown in

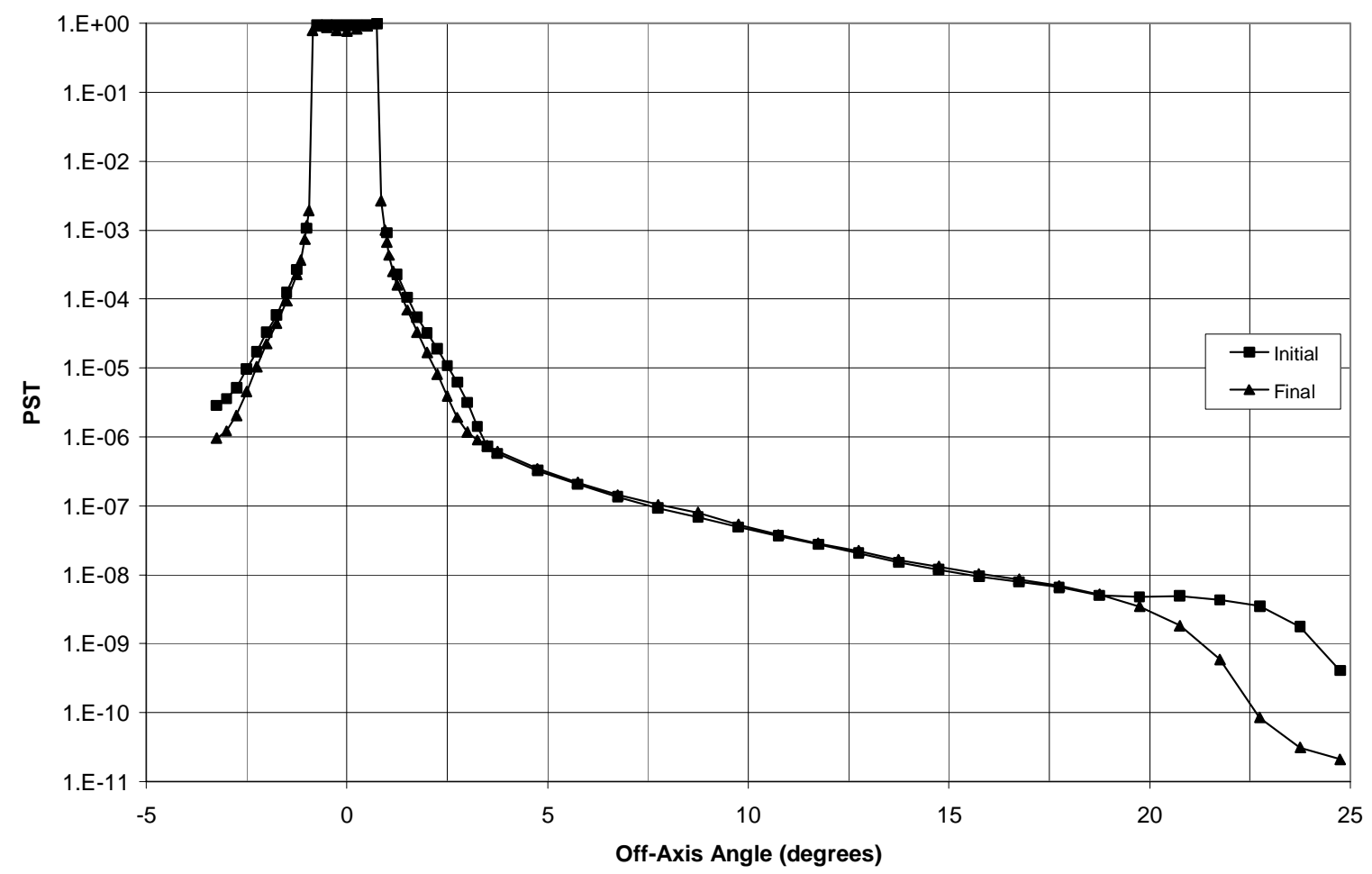

Figure 5. Off-axis response comparison between initial and final measurements. 


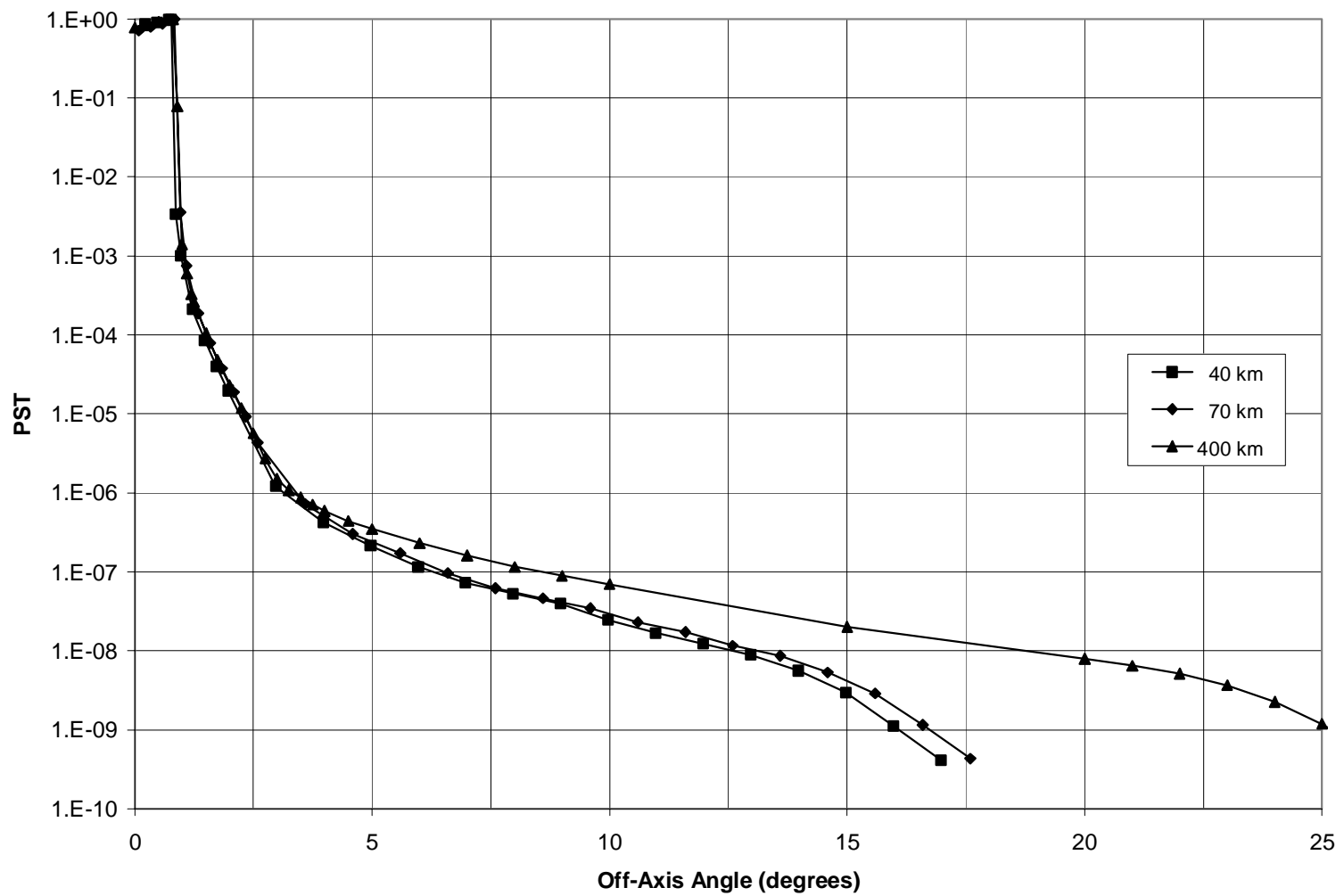

Figure 6. Measured response at tangent heights of $40 \mathrm{~km}, 70 \mathrm{~km}$, and $400 \mathrm{~km}$.

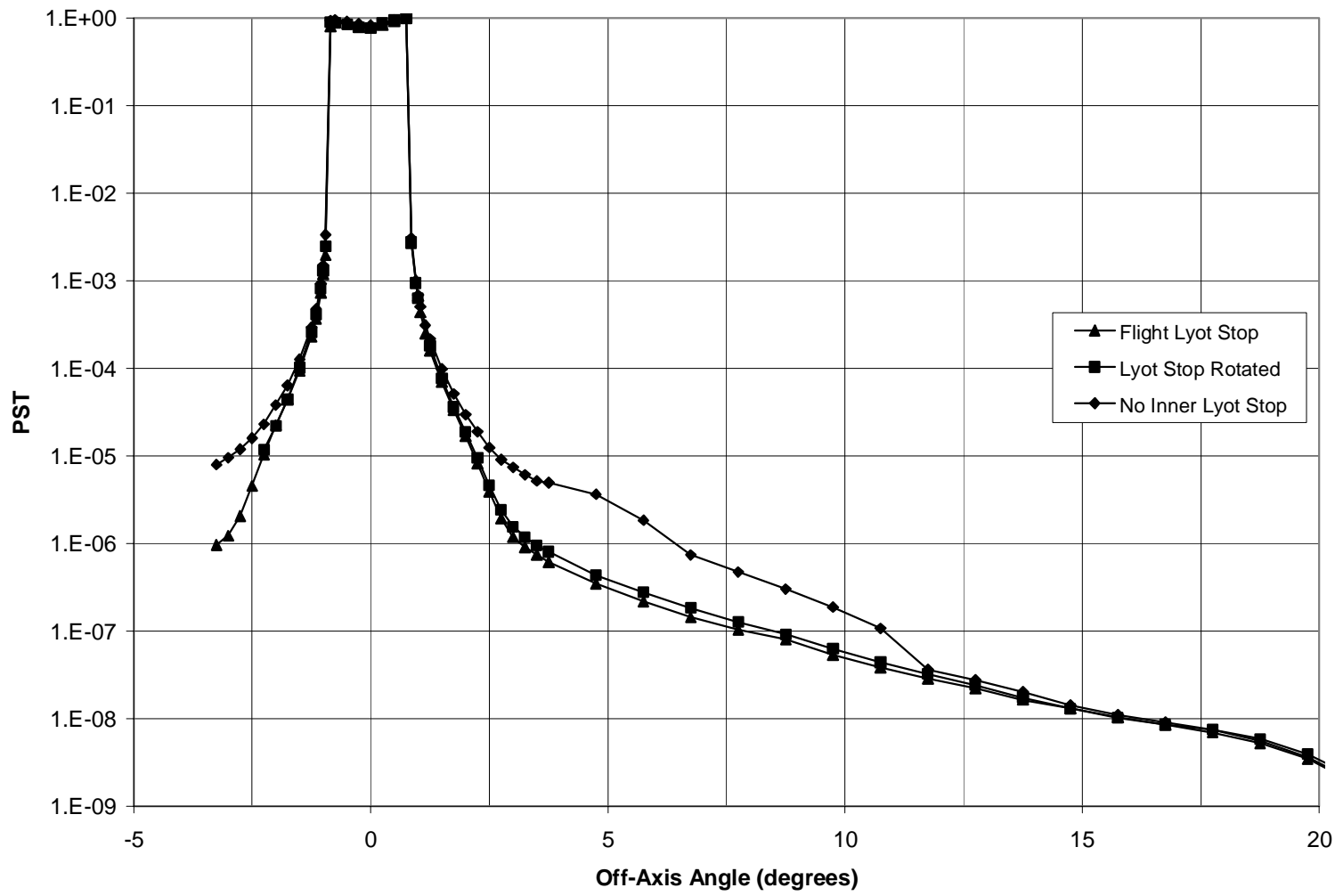

Figure 7. Off-axis responses showing the benefits of using an inner Lyot stop. 
comparison. The "Lyot Stop Rotated" data were taken with the Lyot stop rotated $180^{\circ}$, which prevented the Lyot stop supports from blocking scattered light off the secondary spider edges. For the "No Inner Lyot Stop" response, the flight Lyot stop assembly was replaced with a spare Lyot stop having the inner disk and supports removed. The stray light paths opened up in the absence of the inner stop dominated SABER's response at angles up to $11^{\circ}$. At visible and short IR wavelengths, the stop mainly blocks scattered light from the primary conical baffle and secondary spider surfaces. The benefits are greater at longer wavelengths since the inner Lyot stop suppresses diffraction from the secondary obscuration and spider edges.

\section{COMPARISON OF MEASURED RESULTS TO SOFTWARE MODEL}

The stray light analysis program APART was used to predict SABER's off-axis response and to aid in the telescope design ${ }^{5,6}$. The software model showed that mirror scatter is the dominant source of stray light for the short wavelength channels. A modified version of the model was created for the SABER configuration used in the Black Hole. The quality of the software output is strongly dependent on the accuracy of the mirror BRDFs programmed into it. APART uses the Harvey-Shack BRDF model. The goal BRDF stated in the mirror specification document ${ }^{11}$ at $1.2 \mu \mathrm{m}$, and measured data of the flight mirrors taken at $0.633 \mu \mathrm{m}$ and $1.55 \mu \mathrm{m}$ by Schmitt Measurement Systems (SMS) were considered. The goal BRDF, 4.81E-3 at $0.573^{\circ}$ with a slope of -2.0 , is based on the mirror manufacturer's measurements of previous samples. All the BRDFs were wavelength scaled from their measured or specified wavelengths to $0.4 \mu \mathrm{m}$ to match the PMT peak sensitivity. BRDF scaling is appropriate for super-polished mirrors in pristine condition. The predicted response using the goal BRDF is representative of the results presented at the $\mathrm{CDR}^{12}$. The SMS measured data resulted in a response significantly higher than the goal BRDF response. The APART generated responses are compared to the measured results in Figure 8. With the exception of the contaminated data at angles less the $3^{\circ}$, the measured response is significantly lower than predicted. This indicates that the actual mirror BRDFs are much better than the measured ones and are most likely better than the goal value.

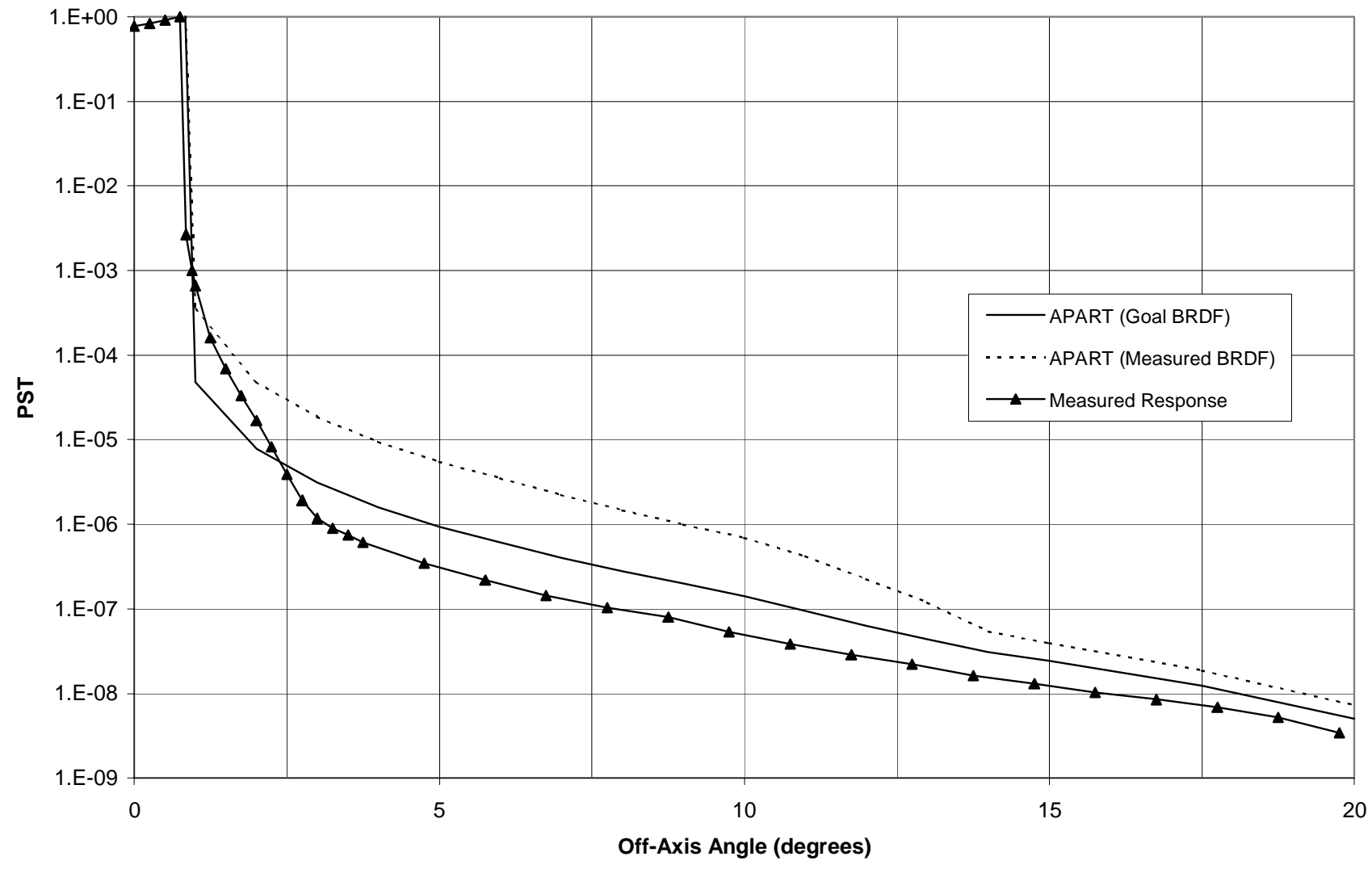

Figure 8. Comparison of predicted and measured off-axis responses. 


\section{REVISED SOFTWARE MODEL USING BRDF ESTIMATES FROM MEASURED RESPONSE}

The BRDF of a mirror is usually determined from measurements made at one or more small areas on the mirror. Multiple measurements are often averaged to estimate the BRDF of the mirror. An alternative approach is to use the off-axis response measurement of a baffled telescope in conjunction with a software model to estimate the BRDF of a single mirror, or an effective BRDF of a system of mirrors. The BRDF of the SABER scan mirror was estimated by analyzing the measured response at off-axis angles from $14^{\circ}$ to $19^{\circ}$. In this region only the scan mirror is directly illuminated by the source, thus is the dominant source of scatter. The BRDF magnitude and slope of the scan mirror was varied in APART until the response most closely matched the measured data. The BRDF at $0.4 \mu \mathrm{m}$ was determined to be $4.10 \mathrm{E}-3$ at $0.573^{\circ}$ with a slope of $-1.46(2.52 \mathrm{E}-4$ at $1.2 \mu \mathrm{m})$. In principle, determination of the primary mirror could be obtained from the measured response from 10 to 14 degrees, the range where only the scan and primary mirrors are illuminated. However, the analysis indicated that the slope of the scan mirror changes with smaller angles, which cast doubt on the primary BRDF fit and subsequent estimation of the secondary mirror BRDF. It was possible to back out an effective BRDF for the mid-angle scan mirror and the fore-optics mirrors by assuming all three have the same BRDF. This does not mean that each mirror contributes the same amount of power to the detector. Using the measured response in the angular range of 4 to 7 degrees, the effective BRDF at $0.4 \mu \mathrm{m}$ was determined to be $1.63 \mathrm{E}-2$ at $0.573^{\circ}$ with a slope of $-2.06(1.93 \mathrm{E}-3$ at $1.2 \mu \mathrm{m})$.

The APART model was revised using the estimated BRDF values stated above. The effective BRDF was assigned to the primary and secondary mirrors for all off-axis source angles. It was also used for the scan mirror at smaller angles. The estimated large angle scan mirror BRDF was applied elsewhere. Figure 9 shows the excellent agreement between the revised APART model and the $400 \mathrm{~km}$ tangent height measured response (lower curves). Furthermore, the model held true when used against the measured $70 \mathrm{~km}$ response (not shown). The figure also shows the measured response of SABER without the inner Lyot stop in comparison to an APART generated response. Since the software model can better represent the flight configuration of SABER, it was utilized to calculate the off-axis response for use in the stray light earth integration routine.

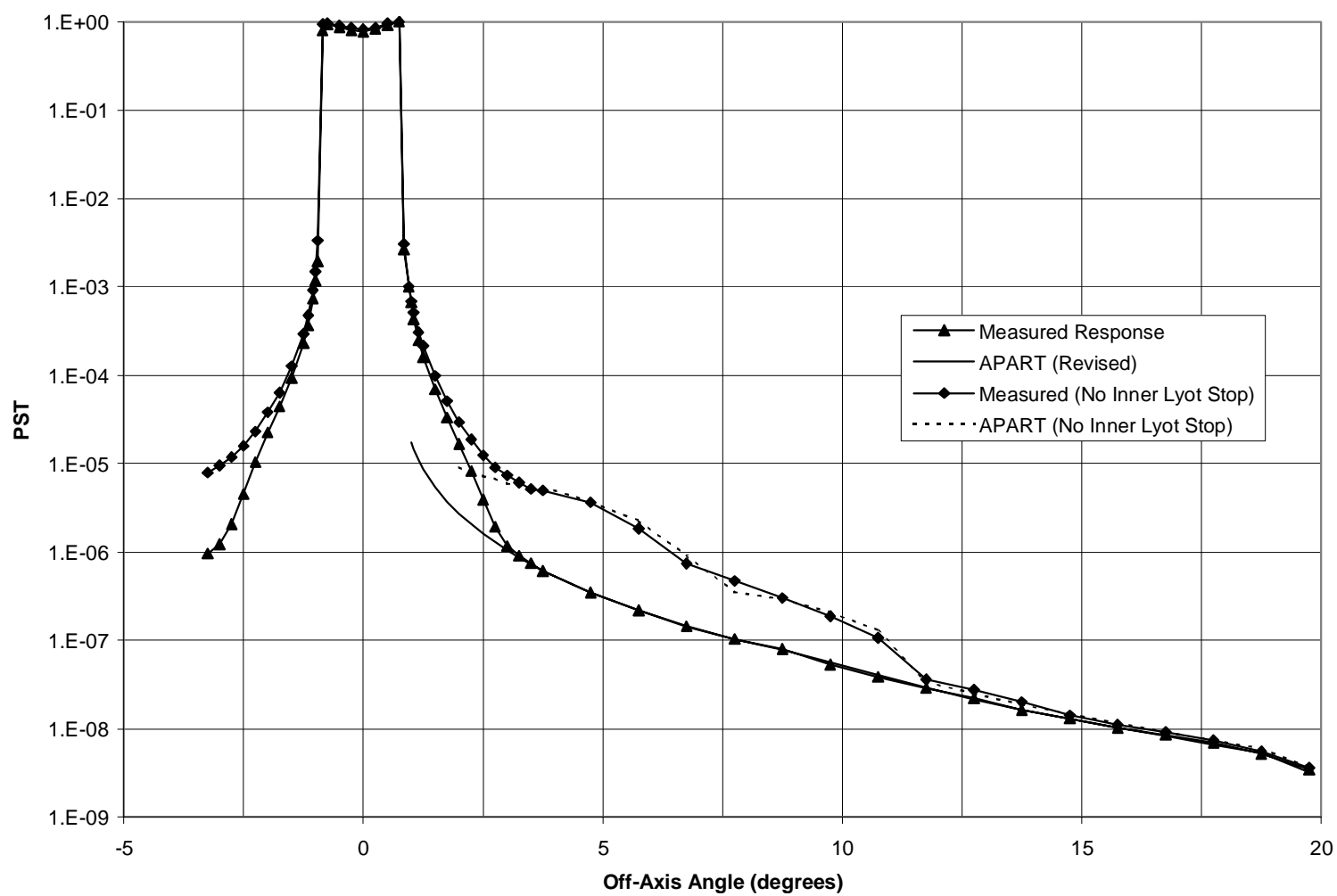

Figure 9. Revised APART response using estimated mirror BRDFs. 


\section{CONCLUSIONS}

The off-axis response measurement of SABER indicates that SABER is an exceptional stray light suppression telescope. No extraneous stray light paths were discovered. The results show that scattered light from the scan, primary, and secondary mirrors dominate the stray light reaching the focal plane in the visible waveband. Although the scan mirror was the main source of stray light, the fore-optics mirrors made a significant contribution at the smaller off-axis angles. The results were better than predicted due to the lower than expected scattering characteristics of the mirrors. The mirror BRDFs were estimated by analyzing the measured response, beginning with the large angle data and working towards the small angle data. These estimates outperformed the goal and measured mirror BRDFs, and were used in a revised APART model to show excellent agreement with the measured response. To conclude, it should be stated that an off-axis response measurement is not meant to replace a software analysis. This paper shows how the two were used to complement each another.

\section{REFERENCES}

1. R. Esplin, C. Batty, M. Jensen, D. McLain, J. Stauder, S. Jensen, C. Stump, D. Robinson, and J. Dodgen, "SABER instrument overview", Infrared Spaceborne Remote Sensing II, Proc. SPIE Vol. 2268, pp. 207-217, 1994.

2. R. Esplin, L. Zollinger, C. Batty, S. Folkman, M. Roosta, J. Tansock, M. Jensen, J. Stauder, J. Miller, M. Vanek, and D. Robinson, "SABER instrument design update", Infrared Spaceborne Remote Sensing III, Proc. SPIE Vol. 2553, pp. 253-263, 1995.

3. “APART stray light analysis software, Version 9.7”, Breault Research Organization 6400 East Grant Road, Suite 350, Tucson Arizona, 85715.

4. “ASAP optical modeling software, Version 5.1”, Breault Research Organization 6400 East Grant Road, Suite 350, Tucson Arizona, 85715.

5. J. Stauder, R. Esplin, L. Zollinger, M. Mlynczak, J. Russell, L. Gordley, and T. Marshall, "Stray light analysis of the SABER telescope", Infrared Spaceborne Remote Sensing III, Proc. SPIE Vol. 2553, pp. 264-270, 1995.

6. J. L. Stauder and R. W. Esplin, "Stray light design and analysis of the Sounding of the Atmosphere using Broadband Emission Radiometry (SABER) telescope," in Infrared Spaceborne Remote Sensing VI, Proc. SPIE, Vol. 3437, pp. 52-59, 1998.

7. G. Peterson, "ASAP analysis of triple diffraction stray light in the SABER system", Breault Research Organization Report No. 3310, February 3, 1998.

8. J.C. Kemp, J.L. Stauder, S. Turcotte and H.O. Ames, "Terrestrial "Back Hole" for measuring high-rejection off-axis response", Infrared Spaceborne Remote Sensing V, Proc. SPIE Vol. 3122, pp. 45-56, 1997.

9. J.L. Stauder, "Off-axis response of SABER as measured in the off-axis scatter facility at the Space Dynamics Laboratory", SDL Document SDL/00-023, 1998.

10. J.L. Stauder, "Stray light comparison of off-axis and on-axis telescopes," Ph.D. dissertation, Utah State University, Logan, UT, 2000.

11. "SABER telescope mirror specifications", Version 1.2, SSG Document, 1998.

12. "SABER critical design review (CDR)", SDL Document SDL/97-076, 1997. 